\title{
Identification and expression analysis of splice variants of mouse enabled homologue during development and in adult tissues
}

Sylvie Veniere1,2, Davy Waterschoot1,2, Joël Vandekerckhove1,2, Anja Lambrechts1,2 and Christophe Ampe*1,2

\begin{abstract}
Background: The Enabled/Nasodilator stimulated phosphoprotein (Ena/NASP) gene family comprises three genes in vertebrates: Vasp, Enabled homologue (Enah) and Ena-VASP like (EVI). Enah has the most complex gene structure. It has extra alternatively included exons compared to Vasp and Evl, and possibly one alternatively excluded intron S. The aim of this mapping study was to probe the occurrence of combinations of exon usage in Enah thereby identifying possible vertebrate ENAH splice variants. We investigated this via an in silico analysis and by performing a reverse transcription-polymerase chain reaction (RT-PCR) screen on mouse samples. We further probed the expression pattern of mouse Enah splice variants during development and in a selection of mouse adult tissues and mouse cell lines.

Results: In silico analysis of the vertebrate Ena/VASP gene family reveals that birds do not have Vasp, while fish have two Evl genes. Analysis of expressed sequence tags of vertebrate Enah splice variants confirms that an Enah transcript without alternative exons is ubiquitously expressed, but yields only limited information about the existence of other possible alternatively spliced Enah transcripts. Via a RT-PCR screen, we provide evidence that during mouse development and in adult mice at least eight and maximally sixteen different Enah transcripts are expressed. We also show that tissues and cell lines display specific expression profiles of these different transcripts. Exons previously associated with neuronal expression of Enah splice variants are also present in other tissues, in particular in heart.

Conclusions: We propose a more uniform nomenclature for alternative exons in Enah. We provide an overview of distinct expression profiles of mouse Enah splice variants during mouse development, in adult mouse tissues and in a subset of mouse cell lines.
\end{abstract}

\section{Background}

The vertebrate Enabled/Vasodilator stimulated phosphoprotein (Ena/VASP) gene family encodes three proteins: enabled homologue (ENAH, throughout the manuscript we use protein, gene and mRNA symbols based on the format of mouse and rat nomenclature, i.e. ENAH, Enah and Enah, respectively) (also referred to as mammalian enabled or Mena), VASP and Ena-VASP like (EVL). Ena/ VASP proteins are actin binding proteins that are involved in dynamic actin remodeling important for maintaining cell shape and cell movement (for review see [1]). These proteins are composed of four conserved domains. An N-terminal Ena/VASP homology domain 1 $(\mathrm{EVH} 1)$ interacts with different proteins and is especially

* Correspondence: christophe.ampe@ugent.be

1 Department of Medical Protein Research, VIB, B-9000 Ghent, Belgium Full list of author information is available at the end of the article important for localization of ENA/VASP proteins in the cell. The central proline-rich region binds to the actin binding protein profilin and Src homology 3 (SH3) and WW domains. The EVH2 domain comprises the globular actin (G-actin) and filamentous actin (F-actin) binding sites and the C-terminal coiled-coil region that mediates oligomerization of the proteins. For VASP no splice variants have been reported, whereas for EVL two splice variants have been discovered: EVL and EVL-I. The latter has an insert sequence in the EVH2 domain [2]. For mouse Enah three alternatively included exons were initially reported: the +++ and ++ exons, located in between exons 3 and 4 , and the + exon that is actually part of an alternative exon 6 due to an upstream splice event $[3,4]$ (Figure 1). Recently, in human Enah, a novel alternatively included exon was found. This exon (11a) is located in between exons 11 and 12 and is at an equivalent position 


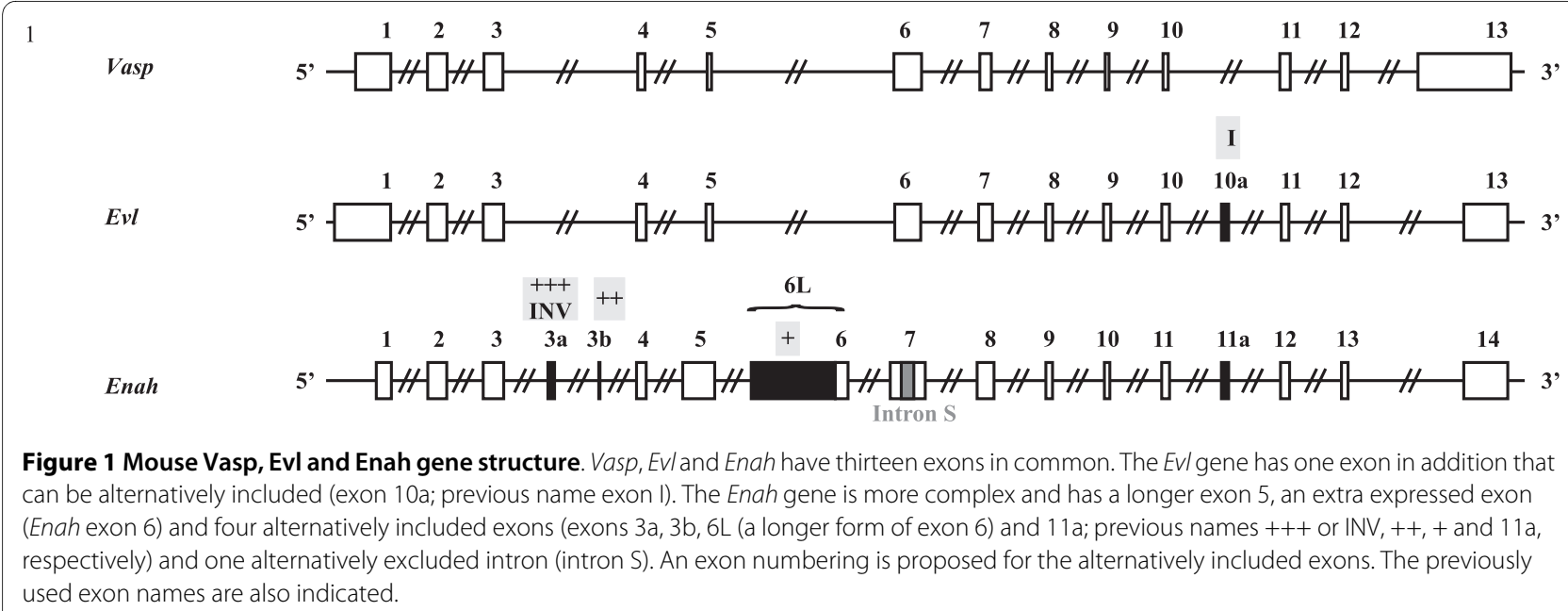

of exon I of mouse $E v l[2,5]$. Next to the alternatively included exons, an alternatively excluded intron has been proposed to exist in mouse Enah. The Enah transcript without this intron (which is part of exon 7) is referred to in literature as Mena short (Mena S) and has no prolinerich region.

ENA/VASP proteins are expressed in various tissues. VASP is highly expressed in platelets (and is the only ENA/VASP protein present there) and is also abundantly expressed in heart, stomach, intestine, spleen, lung and blood vessels [6-8]. ENAH is abundantly expressed in the brain and heart whereas it is not detectable in platelets and spleen $[3,7,8]$. Both mouse VASP and ENAH colocalize in stomach, intestine, kidney and heart [7]. EVL is expressed in the brain but also in spleen, thymus and testis [9].

Neuronal tissue specific expression has been reported for the mouse Enah splice variants containing exons +++ , ++ or $+[3]$. One transcript only contains exon + whereas a second and a third transcript contain exon + combined with either exon ++ or exon +++ . Expression of human Enah transcripts containing the 11a exon is characteristic for epithelial tumor cell lines and this form appears to be involved in proliferation $[5,10]$. This form is down-regulated in invasive tumor cell lines whereas Enah splice variants containing alternative exons ++ and +++ are upregulated $[11,12]$. Furthermore, the splice variant containing exon +++ promotes carcinoma cell motility and invasiveness in vivo and in vitro. This exon was therefore renamed INV (for invasive) [12]. The short Enah splice variant lacking intron $S$ is preferentially expressed in spleen and B-lymphoid cell lines [13]. Also, Evl and the alternative splice variant Evl-I are differentially expressed in tissues. EVL is the predominant form in the brain, whereas EVL-I is enriched in thymus and spleen [2].

From the above studies, it is evident that attempts have been made to document expression of Enah and Evl splice variants. These studies, however, only focused on specific splice variants with only one or maximum two alternatively excluded exons. In general, information on tissue specific expression or expression during development of Enah splice variants is still lacking.

In this study we obtained a more complete view on the spectrum of expressed Enah and Evl splice variants. Next to in silico analysis we validate the existence of the mouse Enah splice variants by expression profiling of these transcripts during development, in tissues and in cell lines. In addition, we propose uniform and consistent names for all exons.

\section{Results}

\section{ENA/VASP phylogeny}

To investigate the general occurrence of the alternative exons described in mouse or human ENA/VASP proteins we first conducted an in silico analysis of ENA/VASP members. To this purpose, we systematically retrieved (predicted) protein sequences from ENSEMBL release 56 [14] and from NCBI [15] for human, mouse, chicken and the model organisms Danio rerio and Xenopus tropicalis. Because a number of these proteins were poorly or incorrectly annotated (hypothetical or by numbers) (see Additional file 1: Supplemental Table S1), we performed a phylogenetic analysis to establish their correct identity (Figure 2). For this analysis orthologues of mouse VASP, EVL and the ENAH splice variants (originally described by Gertler [3]), and selected sequences from the invertebrates Drosophila melanogaster, Strongylocentrotus purpuratus, Hirudo medicinalis, Caenorhabditis elegans and Dictyostelium discoidum were used. This search and the subsequent phylogeny yielded two surprising results. We did not find a VASP orthologue in birds, and fish have two Evl genes (D. rerio (Dr) Evla and Evlb in Figure 2) (see Additional file 1: Supplemental Results). The phylogeny also indicated that ENAH, EVL and VASP arose early in 


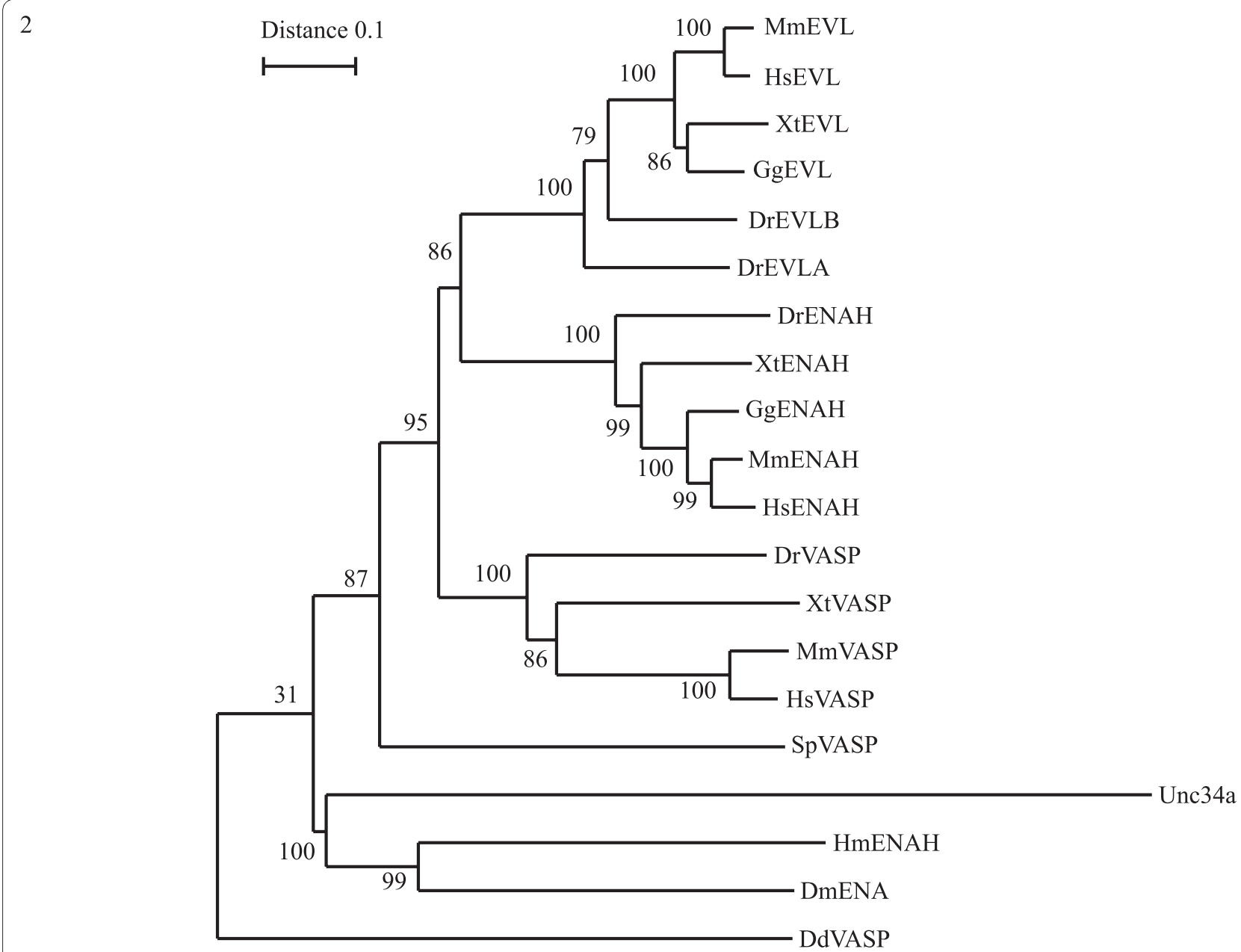

Figure 2 Phylogenetic tree of Ena/VASP homologues. The tree was constructed using the protein sequences in additional file 1: Supplemental Table S1 and was rooted on Dictyostelium discoidum VASP. Hs, Homo sapiens; Mm, Mus musculus; Xt, Xenopus tropicalis; Gg, Gallus gallus; Dr, Danio rerio; Sp, Strongylocentrotus purpuratus; Dd, Dictyostelium discoidum; Hm, Hirudo medicinalis; Dm, Drosophila melanogaster. Bootstrap values in percent are given and indicate high confidence in the vertebrate part of the three.

vertebrate evolution (Figure 2 and additional file 1). ENAH and EVL are more related to each other. Thus the ancestral vertebrate ENA/VASP protein appears to have first given rise to a VASP homologue and an ENAH/EVL ancestor.

\section{ENA/VASP gene structure}

Having established the corresponding ENA/VASP homologues in the selected vertebrates we investigated conservation of their gene structures available in ENSEMBL (see Additional file 1: Supplemental Table S2). In Figure 1, we introduce a more uniform exon nomenclature. The gene structures of vertebrate Enah, Vasp and Evl are largely conserved, as they share thirteen coding exons with, mostly, similar start and end positions and lengths (Figure 1). Exceptions are exon 5, which is much longer in Enah and may encode a coiled coil domain (as predicted by SMART) and Enah exon 6, which is absent in Vasp and
$E v l$. All investigated Vasp forms have this thirteen-exon structure. $E v l$ has fourteen exons because of the presence of the extra alternatively transcribed exon (10a) between exons 10 and 11 . One exception is D. rerio Evl, which appears to have no such exon. This extra alternatively transcribed exon is also present in vertebrate Enah forms (here 11a). Enah forms all contain seventeen exons because two additional alternatively included exons (3a and $3 \mathrm{~b}$ ) are present between exons 3 and 4. Additional transcripts can be generated by an alternative upstream splice event of exon 6, creating the longer exon 6L (exon $6 \mathrm{~L}$ and 6 thus share the 3' part of the exon) (Figure 1). Exon 3 a corresponds to the previously identified exons +++ or INV in mouse and human and exon $3 \mathrm{~b}$ is the ++ exon in mouse. Exon 6L corresponds to the region spanning exon + and exon 6 [3-5]

Usage of the different exons adds amino acids to different parts of the ENAH protein (Additional file 2: Supple- 
mental Figure S1). These may influence ENAH protein function in different ways. By inclusion of peptides encoded by exons $3 \mathrm{a}$ and $3 \mathrm{~b}$, nineteen and four amino acids are respectively inserted between the EVH1 domain and the proline-rich region of ENAH. This may alter the protein binding properties of the EVH1 domain and thus localization of ENAH, or change the relative position of the EVH1 domain with respect to the proline-rich domain. Insertion of exon 6L encodes an ENAH protein with a longer proline-rich region and thus a protein that may bind more profilins and/or multiple different SH3or WW- containing proteins. ENAH with the insertion of the twenty-one amino acids encoded by exon 11a has a longer EVH2 domain. This insert is located between the actin binding motifs $[3,5]$ and the tetramerization domain. Because of a different orientation of the actin binding region relative to the oligomerization domain, the splice variants containing these extra amino acids may have altered actin reorganization properties.

\section{In silico evidence for EVL and ENAH alternative splice variants}

The extra exon 10a in $E v l$ gives raise to two alternative splice variants. TBlastN searches using the protein sequence encoded by exon 10 and 11 with or without the EVL-I sequence encoded by exon 10a, in expressed sequence tag (EST) databases, readily revealed that both transcripts exists in human, mouse, chicken and Хеnориs tropicalis. As mentioned above, there is no evidence from the gene structure for an EVL-I exon in D. rerio and EST searches also proved negative.

The Enah gene structure predicts that multiple splice variants of mammalian ENAH can exist. In the NCBI GenBank, however, only five mouse Enah reference sequences of transcripts are present. Transcript 4 has no alternatively included exons [GenBank: NM 001083121.1] and is usually referred to as the "general" Enah splice variant. Transcript 1 contains exons $3 \mathrm{a}$ and 6L [GenBank: NM 010135.2], transcript 2 has exons $3 \mathrm{~b}$ and 6L [GenBank: NM 008680.2] and, transcript 3 exon 6L [GenBank: NM_001083120.1]. In addition, a form containing exon 11a has been deposited [Genbank: XM 001473812.1]. Similarly, in human, only two reference sequence transcripts are present: transcript 1 containing exon 11a [GenBank: NM 001008493.1], and transcript 2 is the general form [GenBank: NM_018212.4]. Chicken has one reference mRNA sequence containing exons $3 \mathrm{~b}$ and 6L [GenBank: NM 204300.1] and Xenopus (tropicalis and laevis) has two reference mRNA sequences that are very similar and have no alternatively used exons [GenBank: NM 001126543.1 and NM 001092972.1]. In D. rerio the general form [GenBank: NM_001045028.2] is present in addition to an incomplete reference mRNA sequence
[GenBank: NM_001013521.1]. Thus these reference sequences give only marginal support for the use of the alternatively included exons. We further explored their usage by searching the EST database for the presence of vertebrate Enah splice variants (see Additional file 1: Supplemental Table S3). Out of 224 mouse ESTs only one contains both exons $3 \mathrm{a}$ and $3 \mathrm{~b}$, supporting the existence of a transcript containing both of these exons. No EST with solely exon 3a was uncovered whereas we found one EST with only exon $3 \mathrm{~b}$. Of the other investigated organisms none had ESTs with exon 3a and/or 3b except for $X$. tropicalis having one EST with exon 3b. Mouse, human and chicken have, three, two and one ESTs with the exon 5_6L junction, respectively. It is of note that one of the mouse ESTs also contains exon 3b (see Additional file 1: Supplemental Table S3). There is no evidence for such ESTs in X. tropicalis and D. rerio but interestingly in these species we found three [GenBank: EL840056.1, AL961188.2, DC145972.1] and one [GenBank: EE690964.1] ESTs respectively lacking the entire exon 6. A blast search performed with exon 11a on the mouse EST database yielded five hits showing this exon is effectively used. EST searches also revealed that human (sixteen ESTs) and chicken (two ESTs) express an 11a containing form. For Xenopus (tropicalis and laevis) and D. rerio we did not find ESTs containing this exon. In addition, for none of the investigated organisms we found ESTs without intron S. In summary, from the EST databases there are singular observations that exon $3 \mathrm{a}$ is used in conjunction with exon $3 \mathrm{~b}$ and exon $3 \mathrm{~b}$ with exon $6 \mathrm{~L}$, whereas there is no evidence for combinations of $3 a$ and $6 \mathrm{~L}$ or 11a and $6 \mathrm{~L}$. Thus although the vertebrate Enah gene can hypothetically generate 32 alternative transcripts, little information on the potential use of the alternative exons and their combinations is present in these databases.

\section{Expression profiling of mouse Enah transcripts; experimental design and strategy}

To further map which Enah splice variants are expressed, we designed primers that are located on exon-exon boundaries at the three sites where an alternative exon can be included or excluded from the transcript (Figure $3 \mathrm{~A}-\mathrm{B}$, Table 1). When such a forward and a reverse primer are combined in a RT-PCR reaction, amplification can only occur from a mRNA target that contains a certain combination of alternative exons (Figure 3A-B). Whereas this increases specificity it automatically results in primer pairs with different sensitivity. We chose, for each primer pair, to perform reactions under a certain condition (fixed total RNA concentration, fixed number of PCR cycles, see Additional file 3: Supplemental Table S4) where many samples gave a strong specific band and nonspecific amplification occurred as little as possible. 


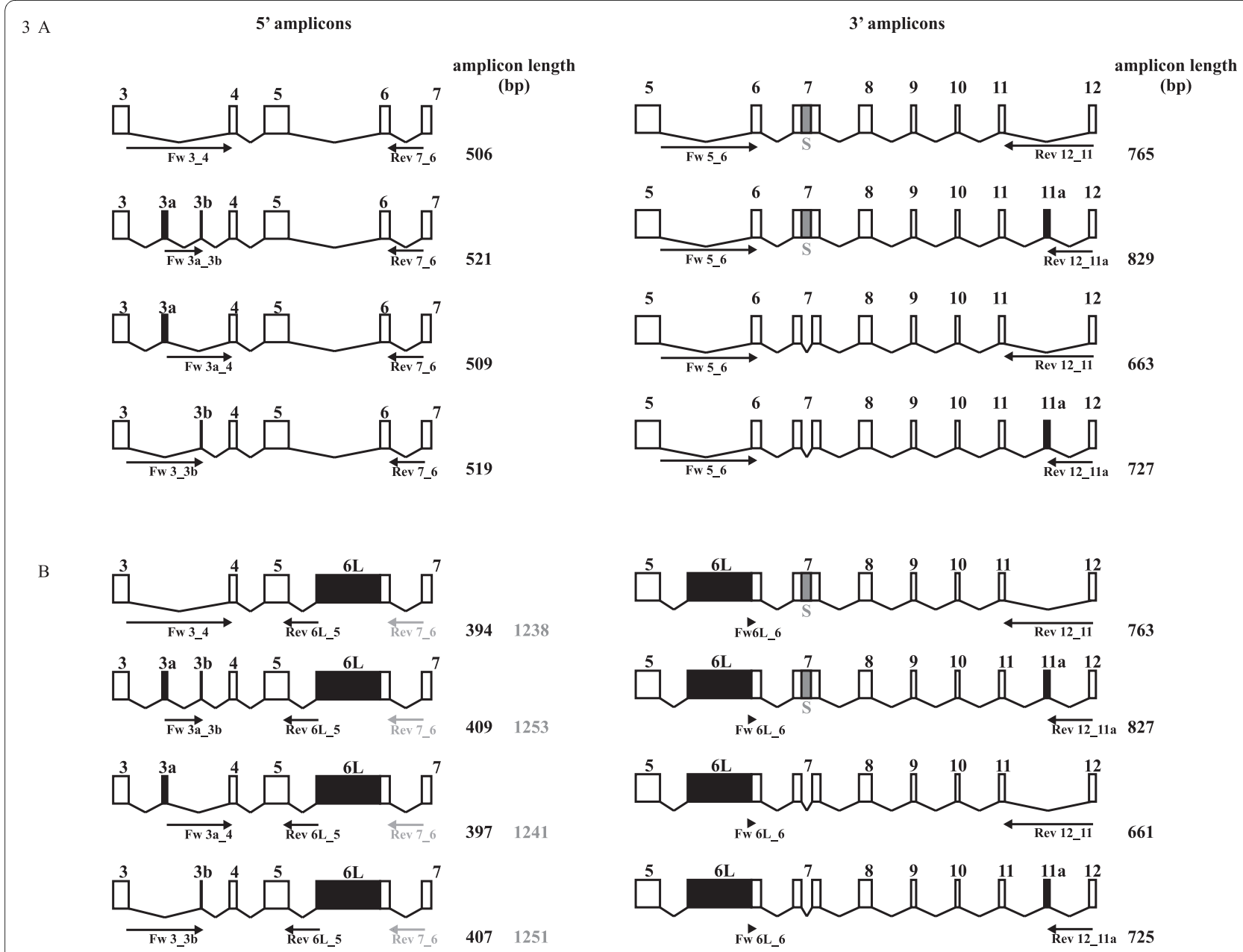

Figure 3 Mouse Enah amplicons. A. Overview of 5' and 3' amplicons with exon 6. B. Overview of 5' and 3' amplicons with exon 6L. (A-B) All theoretical amplicons are shown. Discrimination between different transcripts occurs by choice of primer pair or -when two amplicons can be formed in one RTPCR- by amplicon length (in case of the absence or presence of intron S) via agarose gel electrophoresis.

However, using this approach low abundant transcripts may escape detection in a given sample. Indeed, in some samples, increasing the number of cycles reveals amplicons that were not visible under the chosen condition (Additional file 4: Supplemental Figure S2). Thus, the absence of an amplicon does not necessarily mean that the transcript is not there, but also that it may be below the detection threshold. In fact, we noticed that longer transcripts, for instance from exon-exon boundaries 3 and 4 to exon-exon boundaries 11 or 11a and 12) were difficult to amplify in a reliable manner on all samples (data not shown) whereas the same primers yielded products with primers located on exon 5 or 7 and 6/6L boundaries (see below). Therefore we divided the transcript into two groups of amplicons: 5 ' amplicons and 3 ' amplicons. The $5^{\prime}$ amplicons potentially combine exon-exon boundaries 3_3a_3b_4 and 5_6L/6_7 and the 3' amplicons combine exon-exon boundaries 5_6L/6_7 and 11_11a_12 and eventually provide information on presence of the $S$ intron (102 bp) via the length differences of these amplicons. Because both 5 ' and 3 ' amplicons always have either the 5_6L or the 5_6 exon-exon boundaries, we also obtain information on full length transcripts. An overview of all possible alternative exon combinations of the primers used and of the obtained amplicons is given in Figure 3AB.

\section{Identification of exon 6-containing Enah splice variants}

Theoretically, using this design sixteen different transcripts containing exon 6 (i.e. the shorter form of exon $6 /$ 6L) can be amplified (combinations of four 5 amplicons and four 3' amplicons). To maximize chances of detection, we probed expression of splice variants in a large sample set i.e. developmental stages, in various adult tissues and in cell lines of different origin. We performed four RT-PCRs (RT-PCR 1-4) to detect the four possible $5^{\prime}$ amplicons with exon 6 and two RT-PCRs (RT-PCR 5-6) to detect the four possible 3' amplicons with exon 6 (RT- 
Table 1: Primers used for RT-PCR analysis of Enah transcripts with or without exons 3a, 3b, 6L and/or 11a.

\begin{tabular}{|c|c|c|}
\hline Transcript with (+) or without (-) alternative exons: & Primer name ${ }^{a}$ & Sequence \\
\hline$+3 a$ and $+3 b$ & Fw 3a_3b & 5'-GCGATGTATTITCTGTGTGTTC-3' \\
\hline$+3 a$ and $-3 b$ & Fw 3a_4 & 5'-GCGATGTATTTTCTGTGGGC-3' \\
\hline$-3 a$ and $+3 b$ & Fw 3_3b: & 5'-ATTCACAGGAAGCAGTGTTC-3' \\
\hline$-3 a$ and $-3 b$ & Fw 3_4 & 5'-TTCACAGGAAGCAGGGC-3' \\
\hline \multirow[t]{3}{*}{$+6 \mathrm{~L}$} & Rev 6L_5 & 5'-GTCTGAAGATGGAGCAGC-3' \\
\hline & Rev 7_6 & 5'-GTCCCAAGACAAGGCCC-3' \\
\hline & Fw 6L_6 & 5'-TTCTCCCTCTGCAGCTGC-3' \\
\hline \multirow[t]{2}{*}{$-6 L$} & Rev 7_6 & 5'-GTCCCAAGACAAGGCCC-3' \\
\hline & Fw 5_6 & 5'-AGAATGTCCAATGCTGCTG-3' \\
\hline$+11 a$ & $\operatorname{Rev} 12 \_11 a$ & 5'-GTGTGGATTTGGGTCTGTG-3' \\
\hline$-11 a$ & Rev 12_11 & 5'-TGTGGATTTGGGTCTGGAG-3' \\
\hline
\end{tabular}

aFormat of primer name: direction ( $\mathrm{Fw}=$ forward or Rev = reverse) followed by the numbers of the exon that form the exon boundary on which the primer is located.

PCRs 5 and 6 each can deliver two amplicons derived from transcripts with or without intron S) (Figure 4, details in panel A). Note that in RT-PCR 1 to 4 (with primer Rev 7_6) also a product with exon 6L can be amplified (Figure 3B) but because the elongation time was optimized for the smaller amplicon, solely transcripts with exon 6 were amplified. The specificity of the primers was evident from amplification reactions on MVD7 cells that do not express mouse ENAH (Figure 4D). In addition, all RT-PCRs performed in parallel (either embryonic stages, adult tissues or cell lines) included a water control (no template) and at least one sample in which the reverse transcriptase was omitted (RT-). The quality of the cDNA in each sample is evident from the amplification of glyceraldehyde-3-phosphate dehydrogenase (Gapdh) and 18S ribosomal RNA (Rn18s) (Figure 4B-CD, two bottom panels).

We start by describing the RT-PCR-results for detection of exon 6-containing transcripts expressed in whole embryos of embryonic stages E9.5 to E18.5 and in head and body fractions of E16.5 to E18.5 (Figure 4B). All four 5 ' amplicons and the two 3 ' amplicons containing exon 6 were detected during development. We did not detect the 3' amplicons without intron S. Thus, consistent with EST presence (see above), we confirm the existence of an Enah transcript with both exons $3 \mathrm{a}$ and $3 \mathrm{~b}$ (RT-PCR 2) and the existence of a transcript with exon 11a in mouse (RT-PCR 6). However, we also detected the existence of new transcripts containing either exon 3a (RT-PCR 3) or exon 3b (RT-PCR 4) together with exon 6 . We observed the presence of all transcripts throughout the different stages, except at the latest stages of embryonic development in which some transcripts remain below the detection level under the conditions used (Figures 4 and additional file 4: supplemental Figure S2A, for RT-PCRs 1, 2, 4 and 6 with higher cycle number).

We performed similar RT-PCRs on different mouse adult tissues (Figure 4C). In kidney and heart we detected all four $5^{\prime}$ amplicons and the two $3^{\prime}$ long amplicons. In the other tissues tested (spleen, liver, lung, muscle, brain, uterus, testis and stomach) we only revealed a subset of mouse Enah transcripts. In liver and spleen, only amplicons without alternative exons are barely or not detectable: both the $5^{\prime}$ and 3 ' amplicon in liver and only the $3{ }^{\prime}$ amplicon in spleen (RT-PCR 1 and 5). Repeating RT-PCR 1 with more amplification cycles confirms the 5 amplicon without alternative exons is also present in spleen as expected (Additional file 4: Supplemental Figure S2B). Surprisingly, although transcripts lacking intron $\mathrm{S}$ were shown to be preferentially expressed in spleen and also present in the brain [13], we again did not detect these transcripts in spleen, in the brain nor in any other tissue. Mouse Enah 5' variants without exon $3 \mathrm{a}$ and $3 \mathrm{~b}$ (which is considered to be part of the ubiquitously expressed form), amplified in RT-PCR 1 or with both exon 3a and 3b (RTPCR 2) are detected in most tissues, whereas 5 ' variants with either exon 3a (RT-PCR 3) or exon 3b (RT-PCR 4) show a more restricted and differential expression pattern. The Enah 3' amplicon without exon 11a (RT-PCR 5) (part of the ubiquitously expressed form) is detected in all tissues tested, whereas the Enah 3' amplicon with exon 11a (RT-PCR 6) is not (or hardly) detected in spleen and liver.

We also tested different mouse cell lines for expression of Enah transcripts with exon 6. The $\mathrm{MV}^{\mathrm{D} 7}$ cell line derived from the Mena/VASP knockout is used as a negative control. We screened an embryonic and an adult connective tissue fibroblast cell line (NIH/3T3 and L-929, 


\begin{tabular}{|c|c|c|c|c|c|c|c|c|}
\hline \multirow[t]{2}{*}{$4 \mathrm{~A}$} & \multirow{2}{*}{$\begin{array}{l}\text { RT- } \\
\text { PCR }\end{array}$} & \multirow[t]{2}{*}{ Primer combination } & \multicolumn{4}{|c|}{ Alternative exons } & \multirow{2}{*}{$\begin{array}{c}\text { Intron } \\
\mathrm{s}\end{array}$} & \multirow{2}{*}{$\begin{array}{l}\text { Amplicon length } \\
\text { (bp) }\end{array}$} \\
\hline & & & 3a & $3 \mathrm{~b}$ & $6 \mathrm{~L}$ & 11a & & \\
\hline & 1 & Fw 3_4+ $\operatorname{Rev} 7{ }_{-6}$ & - & - & - & NA & $\mathrm{NA}$ & 506 \\
\hline & 2 & Fw 3a_3b + Rev 7_6 & + & + & - & $\mathrm{NA}$ & $\mathrm{NA}$ & 521 \\
\hline & 3 & Fw 3a_ $4+\operatorname{Rev} 7$ _6 & + & - & - & NA & $\mathrm{NA}$ & 509 \\
\hline & 4 & Fw 3 $33 b+\operatorname{Rev} 7{ }_{6} 6$ & - & + & - & $\mathrm{NA}$ & $\mathrm{NA}$ & 519 \\
\hline & 5 & Fw 5_6 $6+\operatorname{Rev} 12 \_11$ & $\mathrm{NA}$ & NA & - & - & + & $\begin{array}{l}765 \\
663\end{array}$ \\
\hline & 6 & Fw 5_6+ Rev 12_11a & NA & NA & - & + & + & 829 \\
\hline
\end{tabular}
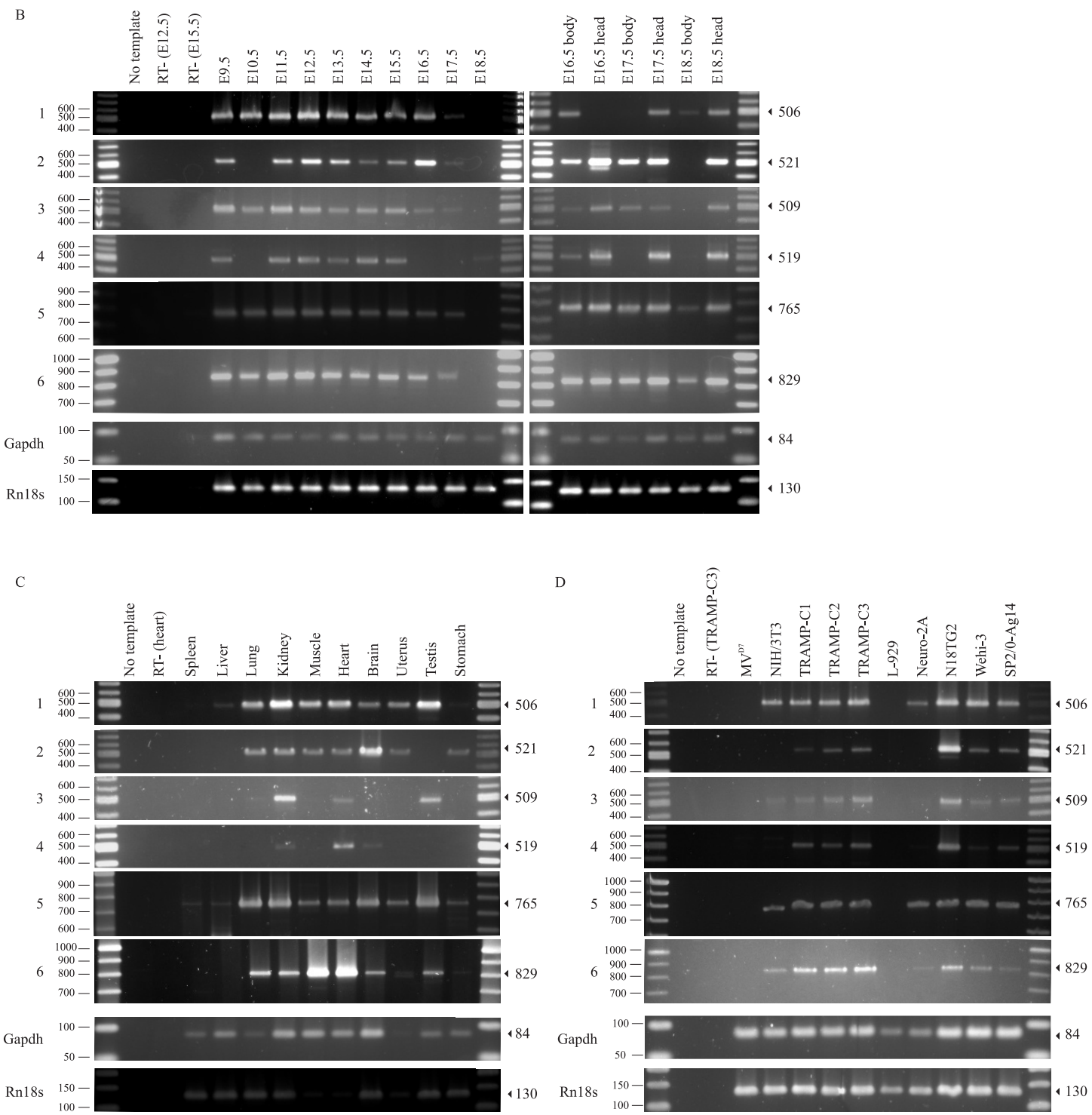

Figure 4 Expression of mouse Enah transcripts with exon 6. A. Details of the RT-PCR reactions: the primers used, the presence $(+)$ or absence $(-)$ of alternative exons and intron and the expected length of the amplicon in base pairs (bp) are indicated. NA, not applicable. B-C-D. The numbers 1-6 of the RT-PCR reactions on the left refer to the primer combinations in panel A. The two bottom panels are the amplifications of the household genes glyceraldehyde-3-phosphate dehydrogenase (Gapdh) and 18S ribosomal RNA (Rn18s). The molecular weight markers (in base pairs) are indicated on the left. The expected size of the amplicon in base pairs is on the right side of the panels. B. Expression of transcripts in whole mouse embryos of day E9.5 to E18.5 and in head and body fractions of E16.5 to E18.5. C. Expression of transcripts in adult mouse tissues. D. Expression of transcripts in the indicated mouse cell lines. MVD7 cells are derived from the Mena/VASP double knockout mouse [27]. 
respectively), two neuroblastoma cell lines (Neuro-2a and N18TG2), the WEHI-3 leukemia cell line, the SP2/0Ag14 myeloma cell line and three epithelial cell lines derived from a primary prostate tumor of the transgenic adenocarcinoma mouse prostate (TRAMP) model [16,17] (Figure 4D). In L-929 cells, no Enah transcripts containing exon 6 were observed (see also Additional file 4: Supplemental Figure S2B). In the NIH/3T3 and the Neuro-2a cell lines we clearly detected the $5^{\prime}$ amplicon with no alternative exons (RT-PCR 1 ) and both 3 amplicons either without (RT-PCR 5) or with (RT-PCR 6) exon 11a. In addition, NIH/3T3 cells express a transcript containing exon 3a (RT-PCR 3). In the TRAMP-C, WEHI-3 and SP2/0-Ag14 cell lines all four 5 ' amplicons and two 3' amplicons with exon 6 are detected. Transcripts without intron $\mathrm{S}$ are again absent.

\section{Mouse Enah splice variants containing alternative exon $6 \mathrm{~L}$}

Alternative exon $6 \mathrm{~L}$ is the largest alternative exon $(843$ bp). This exon is translated to an extra proline-rich sequence located between the LERER region and the central proline-rich region of the ENAH protein. This exon is described to be expressed in the brain [3,18]. As is the case for transcripts with exon 6, sixteen different full length transcripts, with exon $6 \mathrm{~L}$ potentially exist. To get insight into the presence of these transcripts we used a similar setup as in the previous section probing $5^{\prime}$ and $3^{\prime}$ end amplicons separately. In this case, for detection of the four possible 5 amplicons, a reverse primer overlapping exon 6L and exon 5 was used (RT-PCR 7-10), whereas for detection of the four 3 amplicons the forward primer is located in exon $6 \mathrm{~L}$ and overlaps the 5 ' exon boundary of exon 6 (RT-PCR 11-12) (Figure 3B and 5A).

In samples of the developmental stages (Figure $5 \mathrm{~B}$ ) we detected six out of eight theoretical amplicons. This means that alternative exons $3 \mathrm{a}, 3 \mathrm{~b}$ or $3 \mathrm{a}$ and $3 \mathrm{~b}$ can be combined with exon $6 \mathrm{~L}$ and that forms with exon $6 \mathrm{~L}$ and 11a also exist. Again, we could not detect transcripts without intron S (reflected by single amplicons in RTPCR 11 and 12).

In adult mouse tissues (Figure $5 \mathrm{C}$ ) the $5^{\prime}$ amplicon with solely exon 6L (RT-PCR 7) was detected in most tissues, whereas the other 5 amplicons were observed in a more limited set of tissues. This screening partially confirms the reported enrichment of amplicons with exon $6 \mathrm{~L}$ in the brain, as six out of eight amplicons were detected in the brain: 5' amplicons with exon 6L solely (RT-PCR 7), with exon $6 \mathrm{~L}$ and exon 3a (RT-PCR 9), with exon $6 \mathrm{~L}$ and exon 3b (RT-PCR 10), with exon 6L, 3a and 3b (RT-PCR 8 ) or with exon $6 \mathrm{~L}$ and exon 11a (RT-PCR 12). However, expression of exon $6 \mathrm{~L}$ is not limited to the brain. This is particularly evident in heart tissue where these six amplicons with exon $6 \mathrm{~L}$ were also found (Figure 5C). Again, in all tissues tested, transcripts without intron $\mathrm{S}$ were not observed. In spleen and liver no Enah transcripts with exon $6 \mathrm{~L}$ were detected. The other tissues revealed a subset of transcripts containing exon $6 \mathrm{~L}$.

The mouse cell lines were also tested for the expression of splice variants containing exon 6L (Figure 5D). In L929 cells, no Enah transcripts containing exon 6L were observed, which means that L-929 cells do not express detectable levels of Enah (Figure 4-5D). In the neuroblastoma N18TG2 cell line six out of eight amplicons containing exon $6 \mathrm{~L}$ were detected, again amplicons without intron S were not observed. Only a subset of these six amplicons were found for the other cell lines. The $5^{\prime}$ and 3 ' amplicons that contain the alternative exon $6 \mathrm{~L}$ without exons $3 \mathrm{a}, 3 \mathrm{~b}$ and $11 \mathrm{a}$ were, however, discovered in all cell lines (with the exception of L-929 cells). In addition, the splice variant containing the exons $6 \mathrm{~L}$ and $3 \mathrm{a}$ was detected in these cell lines.

\section{Discussion}

Previous expression studies have focused on the ubiquitously expressed form of mouse ENAH. Here we identify new mouse Enah transcripts that have various combinations of alternatively included exons and thus provide a more complete view of the expression profiles of distinct Enah transcripts. This is of significance for studies linking expression of certain Enah exons to proliferation or invasion of tumor cell lines [5,10-12]. Clearly, in these types of informative correlative studies combinations of used exons will have to be taken into account as well.

Protein sequences and the gene structures of vertebrate Ena/VASP homologues are relatively well conserved. Mouse Enah potentially gives rise to 32 different transcripts. An initial in silico analysis of Enah splice variants, considering all species investigated (human and the model organisms mouse, chicken, $D$. rerio and $X$. tropicalis or laevis), provided evidence for the existence of at least one EST containing either exon $3 \mathrm{a}, 3 \mathrm{~b}, 6 \mathrm{~L}$ or $11 \mathrm{a}$ (see Additional file 1: Supplemental Table S3). However, we found no EST evidence for the absence of the alternative intron S. It is worth noting that we could only retrieve a minimal set of ESTs containing these alternative exons. For mouse, we found 224 Enah ESTs of which only nine have alternative exons. This validates that the Enah splice variant without alternative exons is the ubiquitous form. Of these nine ESTs with alternative exons, only four different alternative exon combinations were found. One EST contains both exons 3a and 3b, one combines exons $3 \mathrm{~b}$ and $6 \mathrm{~L}$, two contain only exon $6 \mathrm{~L}$ and five have only exon 11a. Because of the limited length of EST sequences, we could not retrieve information about exon combinations 3a and/or 3b with exon 11a. Moreover, a Genbank search only retrieved five mouse Enah reference sequences, whereas in theory, if all combinations of alternative exons and introns are used, 32 Enah transcripts 


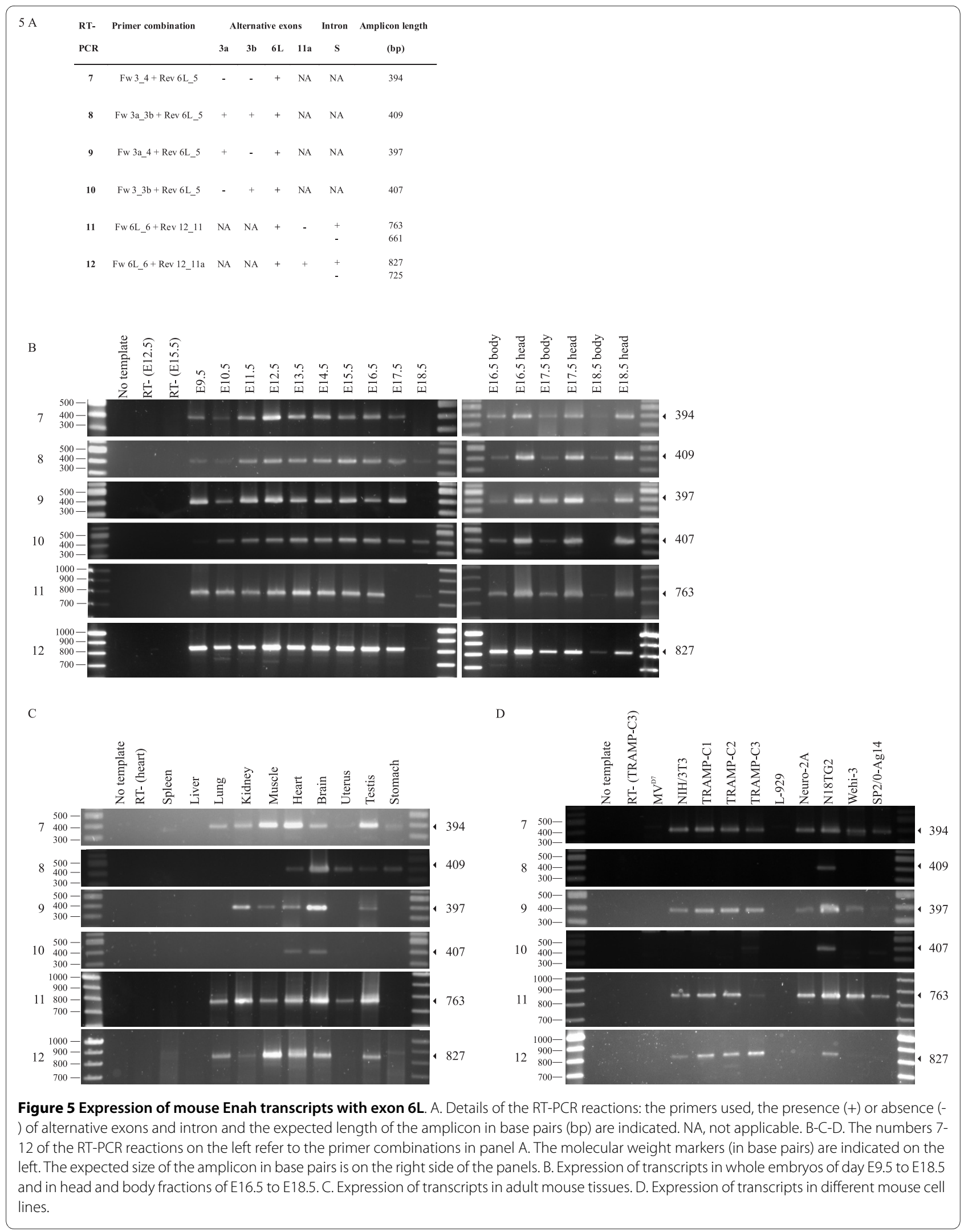


may exist. This indicates that the databases potentially are a poor reflection of the true complexity of the Enah transcript family. We therefore designed a RT-PCR screen to investigate the presence of mouse Enah transcripts during development, in adult tissues and in cell lines. As there might be a strong bias to the ubiquitous Enah splice variant without alternative exons (as suggested by the frequency of its occurrence in EST databases), we probed specifically for sets of transcripts using exon-specific primers. In an initial attempt we tried to amplify long Enah transcripts to get information on all possible exon combinations. We however failed to amplify these products in a reproducible manner from the various samples. Therefore we divided transcripts into two amplicons that overlap at the exons 6 or $6 \mathrm{~L}$. We detected, via this approach, the existence of eight Enah transcripts with different $5^{\prime}$ region exons and four Enah transcripts with different 3 ' region exons (for an overview see Additional file 5: Supplemental Figure S3). This proves that at least eight and maximally sixteen mouse Enah transcripts exist. Until now, expression of variants with exon 11a was only shown in human [5]. Here, we show that mouse transcripts with exon 11a (either in combination with exon 6 or with exon 6L) are expressed in several tissues and cell lines.

Similar to the EST searches, we have no evidence for transcripts without intron $\mathrm{S}$ in mouse. Tani and colleagues discovered mouse Enah transcripts without intron S (in exon 7) using a similar RT-PCR set-up as the one described here [13]. They performed a RT-PCR with a forward primer located at the beginning of exon 7 and a reverse primer located in exon 8 on cDNA of mouse adult brain, spleen and on cDNA of embryonic day 17 . Therefore, an amplicon representative for this splice variant should in principle be present in our RT-PCR assays 5, 6, 11 and 12. We note, however, that in spleen also other Enah transcripts are difficult to detect. It is however unclear why we did not detect this splice variant in E17.5 or in the brain.

ENAH is expressed at high levels in the developing mouse brain where it plays a role in regulation of growth cone dynamics and axon guidance [18-20]. Consistent with this, the screening of mouse embryonic stages shows that all of the identified partial transcripts were found in mouse embryonic stages and in head fractions at E18.5. In adult mice, tissue specificity has been shown for some mammalian ENAH splice variants. Mouse ENAH was detected in several tissues by immunoblotting, but given the aberrant mobility of the protein on sodium dodecyl sulphate polyacrylamide gels and the small size contributed by the coding information in exons $3 a$ (nineteen amino acids) and $3 \mathrm{~b}$ (four amino acids), it is unclear whether such forms can be resolved by this technique. In addition, the fact that few ESTs are present in the data- base suggests these transcripts are not abundantly expressed and thus likely also the protein variants will be rare. Mouse Enah splice variants with exon 6L are distinguishable from Enah splice variants with exon 6 via western blotting and are abundantly expressed in the brain $[3,7,18]$. We confirm that all three described variants with exon $6 \mathrm{~L}$ are detected in the adult brain. In addition, a transcript which has exon 3a, exon $3 \mathrm{~b}$ and exon $6 \mathrm{~L}$ and a transcript containing both exon 6L and exon 11a are present in this tissue. However, we find that exon $6 \mathrm{~L}$ is not exclusively used in the brain. Variants with exon 6L are also present in other tissues in particular in heart. This seems in contrast with earlier findings that ENAH with the extra sequence encoded by exon $6 \mathrm{~L}$ was only detected via western blotting in the brain (and not in heart, kidney, any other tissue tested or in NIH/3T3 cells) $[7,18,21]$. RT-PCR is a more sensitive method and if appropriately designed can also detect low abundant transcripts. ENAH proteins containing the extra sequence encoded by exon $6 \mathrm{~L}$ might be present at levels that are below detection level for western blotting. On the other hand, post-transcriptional regulation might prevent the transcripts from being translated in vivo.

ENAH has been shown to be abundantly expressed in heart and to be important for adult mouse heart function. Indeed, the hearts of Enah knockout mice develop structural and electrical conductivity abnormalities postnatally (unpublished data mentioned in [22]). Also, combined disruption of the localization of ENAH and VASP in cardiac intercalated disks causes dilated cardiomyopathy and early postnatal lethality, whereas VASP knock-out mice show no cardiac abnormalities [8,23,24]. Here we show that all of the discovered Enah transcripts are expressed in mouse adult heart, which suggests important specialized functions for ENAH splice variants in adult heart.

We screened mouse cell lines for Enah splice variants, and found that L-929 fibroblast cells do not detectably express Enah. NIH/3T3 fibroblast cells do, however, express several Enah transcripts, indicating differences exist within cell lines of related origin. A similar difference was shown in [5] in which six human breast tumor cell lines abundantly express ENAH, whereas in one (DAL) it is hardly detectable. All other cell lines tested express more than one Enah variant and N18TG2 cells express all eight transcripts with different 5 ' end and four transcripts with different 3 ' end and thus contain at minimum eight and possibly sixteen Enah transcripts. The three prostate cancer cells are derived from a primary prostate tumor of the transgenic adenocarcinoma mouse prostate (TRAMP) model and are of epithelial origin $[16,17]$. These all three express splice variants with the 11a exon which has been previously correlated with an epithelial phenotype and with proliferation of epithelial 
tumor cell lines [5]. Here, we in addition show that splice variants with the 11a exon are also expressed in cell lines that are not of epithelial origin. This is in line with recent findings from Warzecha and collegues [25]. Although these authors report that Enah with exon 11a is strongly present in human epithelial cells and down-regulated upon induction of epithelial-mesenchymal transition, they also show that this transcript is present at basal levels in MDA-MB-231, a cell line with a mesenchymal phenotype. Splice variants containing exon $3 \mathrm{a}(=\mathrm{INV})$ or $3 \mathrm{~b}$ have been associated with invasion [11,12]. Forms containing this exon are present in the TRAMP C1 and C2 cell lines that are invasive in vitro and tumorigenic when grafted into syngeneic C57BL/6 hosts, but also in the C3 cell line that is non-tumorigenic $[16,26]$. Here, we show that transcripts with exons $3 \mathrm{a}, 3 \mathrm{~b}$ or both are present in all cell lines tested (except for L-929). Expression of splice variants containing exons $3 \mathrm{a}$ or $3 \mathrm{~b}$ (in combination with exon $6 \mathrm{~L}$ ) was shown in the brain indicating that expression of these exons is not limited to invasive cells [3]. We note that Enah transcripts from invasive Polyomavirus Middle T gene mouse transgenic tumors either contained the $3 \mathrm{a}$ or the $3 \mathrm{~b}$ exon but a combination of these two exons was not found [11]. The TRAMP-cell lines do, however, express such forms.

\section{Conclusions}

In summary, we report the expression of mouse Enah splice variants, caused by variable insertion of four alternative included exons (3a, 3b, 6L and 11a), in a qualitative way. By covering all possible exon combinations we provide the first strong evidence of the complexity in the ENA/VASP family on the transcript level. If we take into account all investigated developmental stages and tissues, each of these splice variants has a unique expression pattern. In different cell lines different subsets of splice variants were detected, indicating mouse tissues and cell lines have distinct profiles of Enah transcripts. We demonstrate by our approach that splice variants, identified in specific studies and attributed to specific tissues or processes, display a wider expression profile. These observations will contribute to studies into understanding ENA/ VASP protein function on an organism-wide scale.

\section{Methods}

\section{Cell culture}

The MVD7 cell line was a kind gift of Dr. F. Gertler (Massachusetts Institute of Technology) and was cultured as described [27]. NIH/3T3 embryonic fibroblast cells, SP2/ 0-Ag14 myeloma cells, Neuro-2a and N18TG2 neuroblastoma cells, L-929 adult connective tissue fibroblast cells and WEHI-3 leukemia cells were cultured in Dulbecco's Modified Eagle Medium (DMEM)-glutamax (Invitrogen, Carlsbad, CA) supplemented with penicillin
(100 units/ml) and streptomycin, $100 \mu \mathrm{g} / \mathrm{ml}$ (Invitrogen, Carlsbad, CA) and heat inactivated $10 \%$ fetal bovine serum (FBS) (Hyclone-Perbio; Brackley, UK). Transgenic adenocarcinoma mouse prostate-C (TRAMP-C) cell lines $(\mathrm{C} 1, \mathrm{C} 2, \mathrm{C} 3)$ were obtained from ATCC and cultured in DMEM containing $4 \mathrm{mM} \mathrm{L}$-glutamine, $4.5 \mathrm{~g} / \mathrm{l}$ glucose without sodium pyruvate (Invitrogen, Carlsbad, CA) supplemented with $10 \%$ FBS, $5 \mu \mathrm{g} / \mu$ l bovine insulin (Sigma) and $10 \mathrm{nM} 5$ alpha-androstan-17 beta-ol-3-one (SigmaAldrich, St. Louis, MO).

\section{RNA isolation, Endpoint Reverse transcription polymerase chain reaction (RT-PCR)}

Total RNA was isolated from flash-frozen mouse tissue and embryos (Swiss mice) (RNeasy Midi, Qiagen, Valencia, CA) or from collected cell lines (High Pure RNA Isolation kit) (Roche, Basel, Switzerland) according to the manufacturer's protocol. DNAse I (Qiagen or Roche) treatment was performed at room temperature for 15 min (Qiagen, Valencia, CA) or 20 min (Roche, Basel, Switzerland) depending on the kit used (RNeasy Midi, Qiagen or High Pure RNA Isolation kit, Roche) according to the manufacturer's instructions. First strand DNA synthesis was performed using $2.5 \mu \mathrm{g}$ of RNA using the anchored -oligo $(\mathrm{dT})_{18}$ primer (Transcriptor First Strand complementary DNA (cDNA) Synthesis kit) (Roche, Basel, Switzerland). Reverse transcription-polymerase chain reaction (RT-PCR) was performed in a T3000 thermocycler (Biometra, Göttingen, Germany) with Faststart taq DNA polymerase (1.5 mM $\mathrm{MgCl}_{2}$ ) (Roche, Basel, Switzerland), dNTPs $(200 \mu \mathrm{M})$ (Invitrogen, Carlsbad, $\mathrm{CA})$ and target specific primers $(0.2 \mu \mathrm{M})$. For mouse Enah: target specific primers were designed based on [GenBank: $\quad$ NM 010135.2, NM 008680.2, NM 001083120.1, NM 001083121.1, BE863360.1 and CF553956.1] (Table 1) and conditions for PCR reactions with each primer set were optimized (see Additional file 4: Supplemental Table S4 for reaction conditions). Primers for household genes were designed as published [28]. The amplicons were separated on a $2 \%$ agarose gel or a 4.5\% NuSieve GTG Agarose gel (Cambrex, East Rutherford, NJ) and for each amplicon a representative sample was cloned with the TOPO-TA cloning kit for sequencing (Invitrogen, Carlsbad, CA). RT-PCR product was cloned in $p C R 4-T O P O$ and transformed into TOP10 E .coli cells. Insertion of a PCR fragment causes disruption of the lethal $E$. coli gene $c c d \mathrm{~B}$ and permits growth of positive recombinants. Cycle sequence reactions were performed with the BigDye Terminator v3.1 cycle sequencing kit (Applied Biosystems, Foster City, CA) in the T3000 thermocycler (Biometra, Göttingen, Germany) and analyzed with the 3100 Genetic analyzer (Applied Biosystems, Foster City, CA). 


\section{Phylogeny}

Protein sequences (see Additional file 1: Supplemental Table S1) were aligned with clustalW2 using the default settings $[29,30]$. The resulting alignment file was converted with Forcon $[31,32]$ and distances were calculated with Treecon [33,34] using Poisson correction and bootstrap analysis with 500 samples. A neighbor joining tree was constructed and rooted on the Dictyostelium discoidum VASP sequence. Parts covering the sequences encoded by exons $3 \mathrm{a}$ and $3 \mathrm{~b}$, or $6 \mathrm{~L}$, or $11 \mathrm{a}$ flanked by the sequences encoded by the preceding and the following exon were used for TBlastN searches at NCBI in the reference gene sequence or EST sequence databases with a restriction to human, mouse, chicken (or birds), Xenopus (tropicalis and/or laevis) or zebrafish (or bony fish). In case of searches for 5_6L encoded sequences or sequences lacking the information of intron $\mathrm{S}$ the expectation value was set to 100 and low complexity filtering was not applied (see Additional file 1: Supplemental Table S1, Table S2 and Table S3 and NCBI links). The ENAH domain structure was analyzed using the SMART tool [35].

\section{List of abbreviations}

cDNA: complementary DNA; Dd: Dictyostelium discoidum; Dm: Drosophila melanogaster; DMEM: Dulbecco's Modified Eagle Medium; Dr: Danio rerio; Ena: Enabled; Enah: Enabled homologue; EST: expressed sequence tag; EVH: Ena/VASP homology domain; EVL: Ena-VASP-like; F-actin: filamentous actin; FBS: fetal bovine serum; G-actin: globular actin; Gg: Gallus gallus; Hm: Hirudo medicinalis; Hs: Homo sapiens; INV: invasive; Mena: mammalian Ena; Mena S: Mena short; Mm: Mus musculus; PCR: polymerase chain reaction; RT-PCR: reverse transcription polymerase chain reaction; SH3: Src homology 3; Sp: Strongylocentrotus purpuratus; TRAMPC: Transgenic adenocarcinoma mouse prostate-C; VASP: Vasodilator stimulated phosphoprotein; Xt: Xenopus tropicalis

\section{Additional material}

Additional file 1 Birds lack VASP and Danio rerio has two Evl genes. supplementary results on the phylogeny of EnaNASP proteins including: Supplemental Table S1 with the Accession numbers of protein sequences used in phylogeny, Supplemental Table S2 with Ensembl gene accession numbers of the genes of selected species and supplemental Table S3 with GenBank accession numbers of Enah EST sequences of the selected species and their source.

Additional file 2 Supplemental Figure S1: Hypothetical ENAH protein sequence. ENAH protein sequence with extra sequences (encoded by alternative exon 3a, exon 3b, exon + (part of exon $6 \mathrm{~L}$ ) and exon 11a) (boxed in grey). The exon numbers are indicated above the protein sequence they encode. The sequence encoded by intron $S$ (part of exon 7) is underlined. Amino acids sequences encoded by different exons are in black or blue (alternating). Amino acids in red are encoded by codons that lie on exonexon boundaries.
Additional file 3 Supplemental Table S4: RT-PCR conditions for Enah 5' and 3'amplicons. the table contains an overview of conditions of RTPCR for 5' and 3'amplicons and for the household genes used in this study. Additional file 4 Supplemental Figure S2: RT-PCR 1, 2, 4 and 6 with increased cycle number. for numbering of RT-PCR reactions, see Figure 4. Data were obtained in an analogous manner as in Figure 4 except that the number of amplification cycles was 50 instead of 40 and the amount of CDNA was $100 \mathrm{ng}$. A. Expression of transcripts in whole embryos of day E9.5 to E18.5 B. Expression of transcripts in adult mouse tissues (spleen, liver and brain) and in mouse cell lines (TRAMP-C3 and L-929). The molecular weight markers (in base pairs) are indicated on the left. The expected size of the amplicon in base pairs is on the right side of the panels.

Additional file 5 Supplemental Figure S3: overview of Enah amplicons. overview of RT-PCR data evidencing the presence or absence of the indicated (left) splice variant in mouse embryonic stages, adult tissues and cell lines, panel A. 5' amplicons, panel B. 3' amplicons

\section{Authors' contributions}

S.V. carried out the experimental work and was involved in acquisition of data, experimental design and interpretation of data. A.L. and C.A. were involved in the experimental design and interpretation of data. C.A. performed the in silico analysis. S.V. and C.A. wrote the manuscript. J.V. participated read and approved the final manuscript. D.W. assisted with cloning, sequencing of RT-PCR fragments and gel electrophoresis.

\section{Acknowledgements}

We acknowledge Marleen Van Troys for reading the manuscript. We thank F. B. Gertler (Massachusetts Institute of Technology) for the MVD7 cell line. A.L. was supported by a post-doctoral fellowship of the Fund For Scientific Research Flanders (F.W.O.-Vlaanderen). This work was supported by grants FWOG0133.06 to C.A. and A.L. and GOA- 12051401 to J.V. and C.A.

\section{Author Details}

'Department of Medical Protein Research, VIB, B-9000 Ghent, Belgium and 2Department of Biochemistry, Faculty of Medicine and Health Sciences, Ghent University, Albert Baertsoenkaai 3, B-9000 Ghent, Belgium

Received: 28 December 2009 Accepted: 17 June 2010 Published: 17 June 2010

\section{References}

1. Bear JE, Gertler FB: Ena/VASP: towards resolving a pointed controversy at the barbed end. J Cell Sci 2009, 122(Pt 12):1947-1953.

2. Lambrechts A, Kwiatkowski AV, Lanier LM, Bear JE, Vandekerckhove J, Ampe C, Gertler FB: cAMP-dependent protein kinase phosphorylation of EVL, a Mena/VASP relative, regulates its interaction with actin and SH3 domains. J Biol Chem 2000, 275(46):36143-36151.

3. Gertler FB, Niebuhr K, Reinhard M, Wehland J, Soriano P: Mena, a relative of VASP and Drosophila Enabled, is implicated in the control of microfilament dynamics. Cell 1996, 87(2):227-239.

4. Urbanelli L, Massini C, Emiliani C, Orlacchio A, Bernardi G: Characterization of human Enah gene. Biochim Biophys Acta 2006, 1759(1-2):99-107.

5. Di Modugno F, DeMonte L, Balsamo M, Bronzi G, Nicotra MR, Alessio M, Jager E, Condeelis JS, Santoni A, Natali PG, et al:: Molecular cloning of hMena (ENAH) and its splice variant hMena+11a: epidermal growth factor increases their expression and stimulates hMena+11a phosphorylation in breast cancer cell lines. Cancer Res 2007, 67(6):2657-2665.

6. Reinhard M, Halbrugge M, Scheer U, Wiegand C, Jockusch BM, Walter U: The $46 / 50 \mathrm{kDa}$ phosphoprotein VASP purified from human platelets is a novel protein associated with actin filaments and focal contacts. Embo J 1992, 11(6):2063-2070.

7. Gambaryan S, Hauser W, Kobsar A, Glazova M, Walter U: Distribution, cellular localization, and postnatal development of VASP and Mena expression in mouse tissues. Histochem Cell Biol 2001, 116(6):535-543.

8. Eigenthaler M, Engelhardt S, Schinke B, Kobsar A, Schmitteckert E, Gambaryan S, Engelhardt CM, Krenn V, Eliava M, Jarchau T, et al: Disruption of cardiac Ena-VASP protein localization in intercalated 
disks causes dilated cardiomyopathy. American journal of physiology 2003, 285(6):H2471-2481.

9. Ohta S, Mineta T, Kimoto M, Tabuchi K: Differential display cloning of a novel rat CDNA (RNB6) that shows high expression in the neonatal brain revealed a member of Ena/VASP family. Biochem Biophys Res Commun 1997, 237(2):307-312.

10. Pino MS, Balsamo M, Di Modugno F, Mottolese M, Alessio M, Melucci E, Milella M, McConkey DJ, Philippar U, Gertler FB, et al: Human Mena+11a isoform serves as a marker of epithelial phenotype and sensitivity to epidermal growth factor receptor inhibition in human pancreatic cancer cell lines. Clin Cancer Res 2008, 14(15):4943-4950.

11. Goswami S, Philippar U, Sun D, Patsialou A, Avraham J, Wang W, Di Modugno F, Nistico P, Gertler FB, Condeelis JS: Identification of invasion specific splice variants of the cytoskeletal protein Mena present in mammary tumor cells during invasion in vivo. Clinical \& experimental metastasis 2009, 26(2):153-159.

12. Philippar U, Roussos ET, Oser M, Yamaguchi H, Kim HD, Giampieri S, Wang Y, Goswami S, Wyckoff JB, Lauffenburger DA, et al:: A Mena invasion isoform potentiates EGF-induced carcinoma cell invasion and metastasis. Dev Cell 2008, 15(6):813-828.

13. Tani K, Sato S, Sukezane T, Kojima H, Hirose H, Hanafusa H, Shishido T: Abl interactor 1 promotes tyrosine 296 phosphorylation of mammalian enabled (Mena) by c-Abl kinase. J Biol Chem 2003, 278(24):21685-21692.

14. ENSEMBL release 56 [http://www.ensembl.org]

15. National Center for Biotechnology Information, protein database [http://www.ncbi.nlm.nih.gov/protein]

16. Foster BA, Gingrich JR, Kwon ED, Madias C, Greenberg NM: Characterization of prostatic epithelial cell lines derived from transgenic adenocarcinoma of the mouse prostate (TRAMP) model. Cancer Res 1997, 57(16):3325-3330.

17. Greenberg NM, DeMayo F, Finegold MJ, Medina D, Tilley WD, Aspinall JO, Cunha GR, Donjacour AA, Matusik RJ, Rosen JM: Prostate cancer in a transgenic mouse. Proc Natl Acad Sci USA 1995, 92(8):3439-3443.

18. Lanier LM, Gates MA, Witke W, Menzies AS, Wehman AM, Macklis JD, Kwiatkowski D, Soriano P, Gertler FB: Mena is required for neurulation and commissure formation. Neuron 1999, 22(2):313-325.

19. Giesemann T, Schwarz G, Nawrotzki R, Berhorster K, Rothkegel M, Schluter K, Schrader N, Schindelin H, Mendel RR, Kirsch J, et al:: Complex formation between the postsynaptic scaffolding protein gephyrin, profilin, and Mena: a possible link to the microfilament system. J Neurosci 2003, 23(23):8330-8339.

20. Menzies AS, Aszodi A, Williams SE, Pfeifer A, Wehman AM, Goh KL, Mason CA, Fassler R, Gertler FB: Mena and vasodilator-stimulated phosphoprotein are required for multiple actin-dependent processes that shape the vertebrate nervous system. J Neurosci 2004, 24(37):8029-8038.

21. Jenzora A, Behrendt B, Small JV, Wehland J, Stradal TE: PREL1 provides a link from Ras signalling to the actin cytoskeleton via Ena/VASP proteins. FEBS Lett 2006, 580(12):455-463.

22. Furman C, Sieminski AL, Kwiatkowski AV, Rubinson DA, Vasile E, Bronson RT, Fassler R, Gertler FB: Ena/VASP is required for endothelial barrier function in vivo. $J$ Cell Bio/ 2007, 179(4):761-775.

23. Hauser W, Knobeloch KP, Eigenthaler M, Gambaryan S, Krenn V, Geiger J, Glazova M, Rohde E, Horak I, Walter U, et al: Megakaryocyte hyperplasia and enhanced agonist-induced platelet activation in vasodilatorstimulated phosphoprotein knockout mice. Proc Natl Acad Sci USA 1999, 96(14):8120-8125

24. Aszodi A, Pfeifer A, Ahmad M, Glauner M, Zhou XH, Ny L, Andersson KE, Kehrel B, Offermanns S, Fassler R: The vasodilator-stimulated phosphoprotein (VASP) is involved in CGMP- and CAMP-mediated inhibition of agonist-induced platelet aggregation, but is dispensable for smooth muscle function. Embo J 1999, 18(1):37-48

25. Warzecha CC, Sato TK, Nabet B, Hogenesch JB, Carstens RP: ESRP1 and ESRP2 are epithelial cell-type-specific regulators of FGFR2 splicing. Mol Cell 2009, 33(5):591-601.

26. Kassis J, Moellinger J, Lo H, Greenberg NM, Kim HG, Wells A: A role for phospholipase C-gamma-mediated signaling in tumor cell invasion. Clin Cancer Res 1999, 5(8):2251-2260.

27. Bear JE, Loureiro JJ, Libova I, Fassler R, Wehland J, Gertler FB: Negative regulation of fibroblast motility by Ena/VASP proteins. Cell 2000, 101(7):717-728.
28. Willems E, Mateizel I, Kemp C, Cauffman G, Sermon K, Leyns L: Selection of reference genes in mouse embryos and in differentiating human and mouse ES cells. The International journal of developmental biology 2006, 50(7):627-635.

29. ClustalW2 [http://www.ebi.ac.uk/Tools/clustalw2/

30. Larkin MA, Blackshields G, Brown NP, Chenna R, McGettigan PA, McWilliam $\mathrm{H}$, Valentin F, Wallace IM, Wilm A, Lopez R, et al:: Clustal W and Clustal X version 2.0. Bioinformatics (Oxford, England) 2007, 23(21):2947-2948.

31. ForCon [http://bioinformatics.psb.ugent.be/software/details/ForCon

32. Raes J, Van de Peer Y: Forcon: a software tool for the conversion of sequence alignments. Embnetnews 1999, 6:10-12

33. TREECON [http://bioinformatics.psb.ugent.be/software/details/ TREECON]

34. Van de Peer Y, De Wachter Y: Treecon for Windows: a software package for the construction and drawing of evolutionary trees for the Microsoft Windows environment. Comput Applic Biosci 1994, 10:569-570.

35. SMART [http://smart.embl-heidelberg.de/]

doi: 10.1186/1471-2199-11-45

Cite this article as: Veniere et al., Identification and expression analysis of splice variants of mouse enabled homologue during development and in adult tissues BMC Molecular Biology 2010, 11:45

\section{Submit your next manuscript to BioMed Central and take full advantage of:}

- Convenient online submission

- Thorough peer review

- No space constraints or color figure charges

- Immediate publication on acceptance

- Inclusion in PubMed, CAS, Scopus and Google Scholar

- Research which is freely available for redistribution

Submit your manuscript at www.biomedcentral.com/submit
C) Biomed Central 